\title{
MORTALIDADE POR NEOPLASMAS EM MULHERES EM IDADE REPRODUTIVA - 15 A 49 ANOS - NO ESTADO DE SÃO PAULO, BRASIL, DE 1991 A 1995
}

*N.HAdDAD, M.B. DA SILVA

Trabalho realizado no Instituto Dante Pazzanese de CardiologiaSecretaria de Saúde do Estado de São Paulo, SP.

RESUMO - OBJeTIVO. Descrever a mortalidade feminina por neoplasmas no período reprodutivo ( 15 a 49 anos), no Estado de São Paulo, de 199I a 1995.

Métodos. A listagem dos óbitos, com as causas básicas codificadas pela Classificação Internacional de Doenças, $9^{a}$ Revisão e as estimativas da população feminina, por grupos etários, foram fornecidas pela Fundação SEADE. Foram calculados coeficientes específicos por 100.000 mulheres, as medianas desses coeficientes relativas ao quinquênio e porcentagens de causas básicas por alguns sub-grupos.

Resultados. Nas mulheres mais jovens, de 15 a 29 anos, a mortalidade maior é relativa ao agrupamento "Neoplasma maligno dos tecidos linfático e hematopoiético", seguida de "Neoplasma maligno dos ossos, do tecido conjuntivo, da pele e da mama". Após os 30 anos, passa a ser preponderante este último agrupamento, seguido de "Neoplasma maligno dos órgãos geni- tourinários", de "Neoplasma maligno dos órgãos digestivos e do peritônio" e de "Neoplasma maligno dos tecidos linfático e hematopoiético". As causas básicas específicas com maior mortalidade foram, nas mulheres de 15 a 29 anos, as leucemias mielóide e linfóide e, nas de 30 a 49 anos, em ordem decrescente, o câncer de mama, o câncer de colo e de porção não especificada do útero, o câncer de estômago, 0 câncer de traquéia, brônquios e pulmão e 0 câncer de ovário e de outros anexos do útero.

Conclusózes. Medidas preventivas devem ser intensificadas para diminuição dos fatores de risco, bem como para diagnóstico e tratamento precoce dos neoplasmas, em mulheres em idade fértil, visando a preservar a sua saúde e a evitar possíveis gestaçóes complicadas com essas doenças.

Unitermos: Mortalidade feminina em idade fértil. Neoplasmas. Câncer de mama. Câncer de útero. Câncer de estômago. Leucemias.

\section{INTRODUÇÃo}

As modificações demográficas, socioeconômicas e sanitárias ocorridas no Brasil, nas últimas décadas, transformaram o perfil de morbi-mortalidade da população brasileira, diminuindo a proporção relativa das doenças infecciosas e parasitárias e aumentando a das doenças cronico-degenerativas, principalmente as cardiovasculares e os vários tipos de câncer'. Em 1994, a morta-

$$
\begin{gathered}
\text { *Correspondência: } \\
\text { Nagib Haddad } \\
\text { Instituto Dante Pazzanese de Cardiologia } \\
\text { Av. Dr. Dante Pazzanese, } 500 \text { - 50 andar } \\
\text { Cep: 04012-909 - São Paulo - SP } \\
\text { Tel: (11) 5085-4211 - Fax: (011) 5085-4161 }
\end{gathered}
$$

lidade proporcional pelas doenças cardiovasculares e pelo câncer estavam em primeiro e segundo lugares, respectivamente, em todo o Brasil, com valores mais elevados nas regiões Sul e Sudeste, as mais desenvolvidas do país².

Alguns estudos sobre mortalidade em mulheres, particularizando a idade reprodutiva, têm sido realizados para identificar as principais causas básicas de óbito. Carvalheiro ${ }^{3}$ verificou, em mulheres de 15 a 49 anos de idade, no Município de Ribeirão Preto, SP, entre 1970 - 1974, que as principais causas básicas de óbito (mortalidade proporcional) foram as doenças do apareIho circulatório (24,7\%), seguidas das doenças infecciosas e parasitárias (21,7\%), dos neoplasmas $(13,9 \%)$ e de lesões e envenenamentos (1 I,2\%). Em estudo posterior, após 15 anos, no mesmo município, Carvalheiro e Manço ${ }^{4}$ encontraram, entre 1985-1989, 28,4\% de óbitos devido às doenças do aparelho circulatório, 20,6\% devido a neoplasmas, $14,9 \%$ devido a lesões e envenenamentos e apenas $6,2 \%$ devido às doenças infecciosas e parasitárias.

Laurenti et al. ${ }^{5}$, em estudo de óbitos de mulheres de I0 a 49 anos, no Município de São Paulo, SP, em 1986, encontraram mortalidade proporcional de 23,6\% para doenças do aparelho circulatório, 19,6\% para neoplasmas, 17,9\% para causas externas e 6, $1 \%$ para doenças infecciosas e parasitárias. 
Haddad e Silva ${ }^{6}$, estudando a mortalidade de mulheres de 15 a 49 anos de idade, em todo o Estado de São Paulo, no período de 199| a 1995, verificaram que, nas muIheres mais jovens ( 15 a 34 anos), os coeficientes mais elevados foram os devidos a lesões e envenenamentos (23,9 a 28,9 por 100.000 mulheres). Entretanto, dos 35 aos 49 anos, as duas principais causas básicas de óbito foram as doenças do aparelho circulatório (46,2 a I 42, I por 100.000 mulheres) e os neoplasmas (35, I a I03, I por 100.000 mulheres). As doenças infecciosas e parasitárias ocuparam a oitava posição na escala descendente dos coeficientes e as lesões e envenenamentos passaram para a terceira ou quinta posição. No período de 199| a 1995, houve um total de 71.041 óbitos de mulheres de 15 a 49 anos, com as seguintes mortalidades proporcionais: doenças do aparelho circulatório - 22,8\%; neoplasmas - 17,6\%; lesões e envenenamentos 16,6\%; doenças do aparelho respiratório $7,4 \%$. As doenças infecciosas e parasitárias foram causa básica de óbito em apenas 4,5\% dos óbitos ${ }^{7}$.

Face à importância crescente dos neoplasmas como causas de mortalidade no Brasil e como verificamos acima, também em mulheres na idade reprodutiva, decidimos apresentar, neste artigo, os dados particularizados para agrupamentos e para causas básicas específicas de óbito por câncer, em levantamento realizado para todo o Estado de São Paulo, no período de 199| a 1995, em mulheres de 15 a 49 anos de idade.

\section{Métodos}

A listagem dos óbitos femininos, de 15 a 49 anos de idade, ocorridos de 199| a 1995, em mulheres residentes no Estado de São Paulo, com as características pessoais e os demais dados contidos nos atestados de óbito, a classificação das causa bási- cas em códigos de quatro dígitos da Classificação Internacional de Doenças - 9a Revisão $(\mathrm{CID}-9)^{8}$, bem como as estimativas da população feminina, por grupos etários, para os mesmos anos, foram fornecidas pela Fundação Sistema Estadual de Análise de Dados (Fundação SEADE) . Para a classificação das causas básicas de óbito, foi utilizado, pela Fundação SEADE, o programa "Automated Classification of Medical Entities" (ACME)9, o qual é um sistema computadorizado que incorpora a estrutura de códigos da Classificação Internacional de Doenças, as regras de seleção e modificação, as relações etiológicas entre os diagnósticos, selecionando automaticamente a causa básica de morte. As estimativas das populações, nos anos intercensitários, foram realizadas considerando-se o crescimento observado no período de $199 \mid$ a 1996, com base na população recenseada em 1991 e na contagem populacional de 1996 e o saldo vegetativo calculado a partir das estatísticas de óbitos e nascidos vivos, processados pela Fundação SEADE.

Os coeficientes específicos de mortalidade foram calculados dividindo-se o número de óbitos por determinadas causas, em determinado grupo etário, pela população feminina do mesmo grupo etário, para o mesmo ano de ocorrência e multiplicando-se por 100.000.

Para a comparação dos coeficientes específicos de mortalidade ocorridos em todo o período de 199| a 1995, em relação aos grupos etários, foram calculados os coeficientes medianos do quinquênio, isto é, foram colocados os cinco coeficientes em ordem crescente e calculadas as medianas.

Para a comparação de causas básicas de óbito dentre as classificadas nos agrupamentos da CID-9, foram feitos cálculos de porcentagem em relação aos totais destes agrupamentos.

A importação dos dados fornecidos em disquete, a contagem das causas básicas de óbito pelos grupos etários e o cálculo dos respectivos coeficientes de mortalidade foram realizados utilizando-se o programa SPSS for Windows, versão 6.0 $0^{10}$.

\section{Resultados}

$\mathrm{Na}$ Tabela I, estão apresentados os coeficientes de mortalidade, por 100.000 mulheres, de acordo com os agrupamentos da CID-9. Os dados não mostram haver tendência crescente ou decrescente, em relação ao tempo, no curto período de cinco anos. Analisando-se os coeficientes medianos para todo o quinquênio, verificamos que os cinco principais agrupamentos de causas básicas, excluindo os sem especificação de localização, em ordem decrescente de grandeza dos coeficientes, foram: "Neoplasma maligno dos ossos, do tecido conjuntivo, da pele e da mama" $(7,45)$, "Neoplasma maligno dos órgãos genitourinários "(5,67), "Neoplasma maligno dos órgãos digestivos e do peritônio" $(5,09)$, "Neoplasma maligno dos tecidos linfático e hematopoiético" $(2,76)$ e "Neoplasmas malignos dos órgãos respiratórios e intratorácicos ( I,32).

Os coeficientes medianos, por grupos etários, para esses agrupamentos de causas básicas de óbito, estão apresentados na Tabela 2, onde verificamos que, dos 15 aos 29 anos, os neoplasmas malignos dos tecidos linfático e hematopoiético mostram os maiores coeficientes, seguidos dos neoplasmas malignos dos ossos, do tecido conjuntivo, da pele e da mama e dos neoplasmas malignos dos órgãos digestivos e do peritônio. Entretanto, a partir dos 30 anos, em ordem decrescente, excluindo-se os "Neoplasmas malignos sem especificação de localização", os maiores coeficientes passam a ser os agrupamentos. "Neoplasmas malignos dos ossos, do tecido conjuntivo, da pele e da mama", "Neoplasmas malignos dos órgãos genitourinários", "Neoplas- 
Tabela I - Coeficientes de mortalidade por neoplasmas (por 100.000 mulheres de 15 a 49 anos), segundo causas básicas (agrupamentos da CID-9) , no Estado de São Paulo, 199I a 1995.

\begin{tabular}{ccccccc}
$\begin{array}{c}\text { Agrupamento } \\
\text { CID-9 * }\end{array}$ & $\mathbf{1 9 9 1}$ & $\mathbf{1 9 9 2}$ & $\begin{array}{c}\text { Anos } \\
\mathbf{1 9 9 3}\end{array}$ & $\mathbf{1 9 9 4}$ & $\mathbf{1 9 9 5}$ & $\begin{array}{c}\text { Coeficiente } \\
\text { mediano }\end{array}$ \\
\hline $140-149$ & 0,29 & 0,33 & 0,38 & 0,43 & 0,34 & 0,34 \\
$150-159$ & 5,03 & 5,09 & 5,03 & 5,58 & 5,34 & 5,09 \\
$160-165$ & 1,40 & 1,26 & 1,20 & 1,74 & 1,32 & 1,32 \\
$170-175$ & 7,01 & 7,45 & 7,42 & 8,29 & 7,68 & 7,45 \\
$179-189$ & 5,67 & 6,26 & 5,60 & 5,57 & 5,68 & 5,67 \\
$190-195$ & 1,25 & 0,95 & 1,31 & 1,36 & 1,52 & 1,31 \\
199 & 1,99 & 1,63 & 1,81 & 1,62 & 1,74 & 1,74 \\
$200-208$ & 2,69 & 2,76 & 3,14 & 2,66 & 2,86 & 2,76 \\
$210-229$ & 0,10 & 0,23 & 0,11 & 0,18 & 0,07 & 0,11 \\
$235-238$ & 0,17 & 0,12 & 0,12 & 0,06 & 0,14 & 0,12 \\
239 & 1,27 & 1,17 & 1,18 & 1,08 & 0,96 & 1,17 \\
\hline
\end{tabular}

* | 40- 149 = Neoplasma maligno do lábio, da boca e da faringe

150-159 = Neoplasma maligno dos órgãos digestivos e do peritônio

160-165 = Neoplasma maligno dos órgãos respiratórios e intratorácicos

170- 175 = Neoplasma maligno dos ossos, do tecido conjuntivo, da pele e da mama

179-189 = Neoplasma maligno dos órgãos genitourinários

190-195 = Neoplasma maligno de outras localizações

199 = Neoplasma maligno sem especificação de localização

200-208 = Neoplasma maligno dos tecidos linfático e hematopoiético

210-229 = Neoplasma benigno

235-238 = Neoplasma de comportamento incerto

239 = Neoplasma de natureza não especificada

Obs. Foram econtrados apenas 2 óbitos tendo "Carcinoma in situ " (230-234)

como causa básica, em todo o período estudado.

mas malignos dos órgãos digestivos e do peritônio", "Neoplasmas malignos dos tecidos linfático e hematopoiético" e "Neoplasmas malignos dos órgãos respiratórios e intratorácicos".

No agrupamento "Neoplasmas malignos dos órgãos digestivos e do peritônio", no período total de cinco anos, para todas as mulheres de 15 a 49 anos de idade, houve 2.376 óbitos, dos quais $30,6 \%$ foram devidos a neoplasma maligno do estômago, 18,6\% a neoplasma maligno do cólon, $12,9 \%$ a neoplasma maligno do fígado e das vias biliares intra-hepáticas, 9,6\% a neoplasma maligno do reto, da junção reto- sigmóide e ânus, 8,5\% a neoplasma maligno do pâncreas e $19,8 \%$ às demais causas.

$\mathrm{Na}$ Tabela 3, estão apresentados os coeficientes medianos, por $100.000 \mathrm{mu}$ Iheres, relativos ao quinquênio, para as causas básicas de óbito específicas, codificadas em três dígitos da CID-9, do agrupamento "Neoplasmas malignos dos órgãos digestivos e do peritônio", segundo os grupos etários. Verificamos que os coeficientes, para todas as causas aumentam com o avançar da idade e que o câncer de estômago prepondera em todas as idades, seguido do câncer do cólon, do câncer do fígado e das vias biliares intra-hepáticas, do câncer do pâncreas e do câncer do reto, da junção reto-sigmóide e ânus.

Houve 628 óbitos devido ao agrupamento "Neoplasmas malignos dos órgãos respiratórios e intratorácicos", no quinquênio, para todas as mulheres, sendo $81,5 \%$ deles causados por neoplasma maligno da traquéia, dos brônquios e do pulmão, 9,4\% por neoplasma maligno da laringe e $9,1 \%$ por outras causas. Na Tabela 4 , verificamos que os coeficientes relativos às duas primeiras causas aumentam mais rapidamente após os 35 anos de idade. 
Tabela 2 - Coeficientes medianos de mortalidade por neoplasmas, segundo os grupos etários e agrupamentos da CID- 9 (por 100.000 mulheres de I5 a 49 anos), no Estado de São Paulo, 199I a 1995.

\begin{tabular}{|c|c|c|c|c|c|c|c|}
\hline \multirow{2}{*}{$\begin{array}{l}\text { Agrupamento } \\
\text { CID-9 }\end{array}$} & \multicolumn{6}{|c|}{ Idade } & \multirow[b]{2}{*}{45 a 49} \\
\hline & 15 a 19 & 20 a 24 & 25 a 29 & 30 a 34 & 35 a 39 & 40 a 44 & \\
\hline |40-149 & 0,06 & 0,06 & 0,20 & 0,07 & 0,47 & 0,91 & 1,56 \\
\hline $150-159$ & 0,13 & 0,68 & 1,67 & 3,52 & 6,59 & 11,58 & 21,25 \\
\hline $160-165$ & 0,12 & 0,13 & 0,33 & 0,68 & 1,52 & 3,58 & 6,12 \\
\hline $170-175$ & 0,97 & 0,84 & 1,79 & 4,80 & 11,07 & 18,88 & 29,58 \\
\hline $179-189$ & 0,34 & 0,68 & 1,55 & 4,00 & 7,52 & 13,46 & 23,55 \\
\hline 190-195 & 0,62 & 0,58 & 0,54 & 0,81 & 1,40 & 2,32 & 3,80 \\
\hline 199 & 0,27 & 0,33 & 0,60 & 1,22 & 2,09 & 3,97 & 7,39 \\
\hline $200-208$ & 1,79 & 1,97 & 2,12 & 2,41 & 3,20 & 4,16 & 6,33 \\
\hline $210-229$ & 0,00 & 0,00 & 0,07 & 0,21 & 0,07 & 0,32 & 0,37 \\
\hline $235-238$ & 0,06 & 0,07 & 0,07 & 0,14 & 0,23 & 0,09 & 0,13 \\
\hline 239 & 0,48 & 0,60 & 0,73 & 0,95 & 1,24 & 2,14 & 2,84 \\
\hline
\end{tabular}

140 - 149 = Neoplasma maligno do lábio, da boca e da faringe

150 - 159 = Neoplasma maligno dos órgãos digestivos e do peritônio

160 - 165 = Neoplasma maligno dos órgãos respiratórios e intratorácicos

170 - 175 = Neoplasma maligno dos ossos, do tecido conjuntivo, da pele e da mama

179 - 189 = Neoplasma maligno dos órgãos genitourinários

190 - 195 = Neoplasma maligno de outras localizações

$199=$ Neoplasma maligno sem especificação de localização

$200-208=$ Neoplasma maligno dos tecidos linfático e hematopoiético

$210-229=$ Neoplasma benigno

$235-238=$ Neoplasma de comportamento incerto

$239=$ Neoplasma de natureza não especificada

Obs. Foram encontrados apenas dois óbitos tendo "Carcinoma in situ " (230-234) como causa básica em todo o período estudado.

Do total de 3.45 I óbitos devido ao agrupamento "Neoplasmas malignos dos ossos, do tecido conjuntivo, da pele e da mama", ocorridos no quinquênio, 84,5\% foram codificados como câncer de mama, 5,0\% como melanoma maligno da pele, 4,9\% como neoplasma maligno dos ossos e das cartilagens articulares, 4,4\% como neoplasma maligno do tecido conjuntivo e de outros tecidos moles e 1,2\% como outros neoplasmas malignos da pele.

Na Tabela 5, observamos que o câncer de mama apresenta coeficientes que aumentam intensamente com a idade a partir dos 30 anos.
No quinqüênio, ocorreram 2.614 óbitos atribuídos ao agrupamento "Neoplasmas malignos dos órgãos genitourinários", sendo 44,5\% codificados como neoplasma maligno do colo do útero, 23,6\% como neoplasma do útero, porção não especificada, 20,8\% como neoplasma maligno do ovário e de outros anexos do útero, 3,9\% como neoplasma maligno do rim, de outros órgãos urinários e de órgãos urinários não especificados e 7,2\% pelas demais causas.

$\mathrm{Na}$ Tabela 6, observamos a elevação dos coeficientes com a idade, mais intensamente para o câncer do colo do útero, câncer do útero porção não especificada e câncer de ovário e de outros anexos do útero. Dos 15 aos 24 anos, o câncer de ovário e de outros anexos do útero apresentaram os maiores coeficientes. Porém, após os 25 anos, os coeficientes de câncer do colo uterino e de útero porção não especificada passam a ser preponderantes, passando o câncer de ovário e de outros anexos do útero para a terceira posição.

No período estudado, ocorreram I.285 óbitos atribuidos às causas do agrupamento "Neoplasmas malignos dos tecidos linfático e hematopoiético", sendo $38,7 \%$ codificados como leucemia mielóide, 20,0\% como 
Tabela 3 - Coeficientes medianos de mortalidade por neoplasmas malignos dos órgãos digestivos e do peritônio, segundo os grupos etários e causas básicas (três dígitos), por 100.000 mulheres de I5 a 49 anos, no Estado de São Paulo, 199 I a 1995.

\begin{tabular}{|c|c|c|c|c|c|c|c|}
\hline \multirow{2}{*}{$\begin{array}{l}\text { Código } \\
\text { CID - 9* }\end{array}$} & \multicolumn{7}{|c|}{ Idade } \\
\hline & 10 a 17 & $\angle 0 \mathrm{~d} \angle 4$ & $2 J$ d $\angle 7$ & & Jग व J7 & $40 \mathrm{~d} 44$ & $45 \mathrm{~d} 47$ \\
\hline 150 & 0,00 & 0,00 & 0,00 & 0,07 & 0,31 & 0,87 & 1,14 \\
\hline$|5|$ & 0,06 & 0,20 & 0,61 & 1,29 & 1,80 & 3,53 & 6,02 \\
\hline 152 & 0,00 & 0,00 & 0,00 & 0,00 & 0,00 & 0,00 & 0,12 \\
\hline 153 & 0,00 & 0,14 & 0,34 & 0,68 & 1,12 & 2,21 & 3,10 \\
\hline 154 & 0,00 & 0,13 & 0,13 & 0,43 & 0,78 & $|| \mid$, & 1,90 \\
\hline 155 & 0,00 & 0,19 & 0,26 & 0,29 & 0,75 & 2,11 & 2,48 \\
\hline 156 & 0,00 & 0,00 & 0,00 & 0,15 & 0,16 & 0,71 & 1,48 \\
\hline 157 & 0,00 & 0,00 & 0,07 & 0,27 & 0,49 & 0,81 & 2,61 \\
\hline 158 & 0,00 & 0,07 & 0,00 & 0,07 & 0,16 & 0,18 & 0,37 \\
\hline 159 & 0,00 & 0,06 & 0,20 & 0,29 & 0,42 & 0,91 & 1,24 \\
\hline \multicolumn{8}{|c|}{ 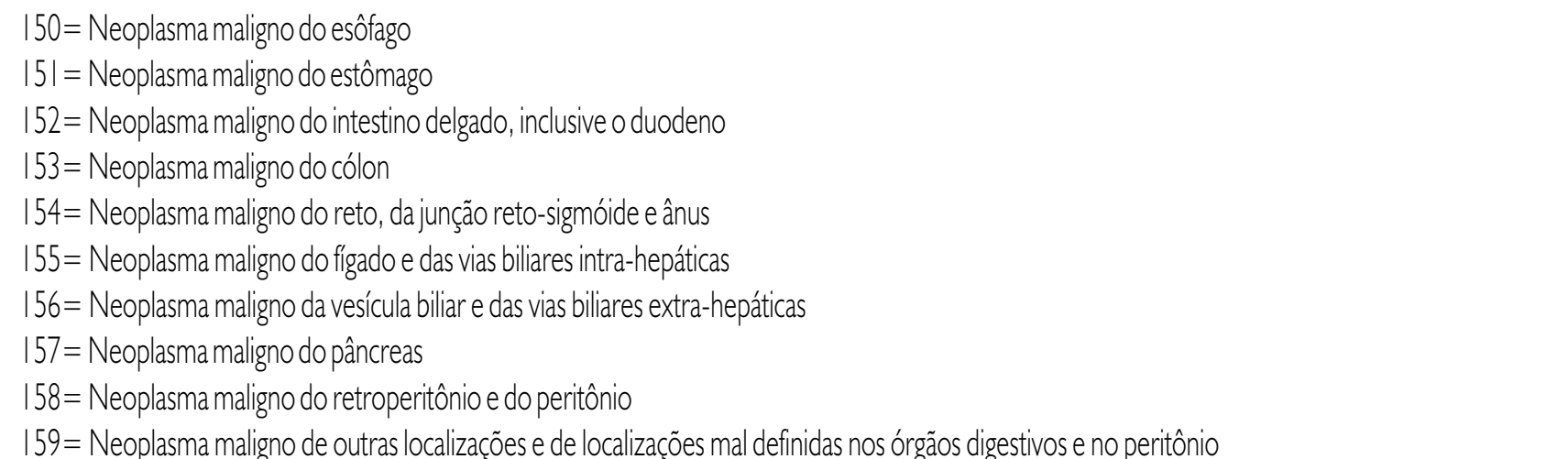 } \\
\hline
\end{tabular}

outros neoplasmas malignos dos tecidos linfóide e histiocitário, 13,0\% como leucemia linfóide, 10,7\% como Doença de Hodgkin, $7,4 \%$ como leucemia de tipo celular não especificado, 6,5\% como mieloma múltiplo e neoplasmas imunoproliferativos e 3,7\% pelas demais causas.

Analisando a Tabela 7, verificamos que o coeficiente de mortalidade por leucemia linfóide, levemente predominante no grupo etário de 15 a 19 anos, vai decrescendo até os 30-34 anos, voltando a se elevar a partir dos 35 anos. Por outro lado, a leucemia mielóide se eleva, paulatinamente, com a idade e é o mais elevado do agrupamento, a partir de 20 anos.

Na Figura I, estão representados os coeficientes medianos de mortalidade, para o quinquênio estudado, das seis principais causas básicas de óbito, por neoplasma maligno, nas mulheres de 15 a 49 anos de idade, segundo os grupos etários. Analisando o gráfico, vemos que os coeficientes de câncer de mama são os mais altos, aumentam com a idade, mais intensamente após os 30 anos. Em segundo lugar, estão os coeficientes de câncer do colo uterino, os quais aumentam também com a idade, porém de forma menos intensa que o câncer de mama. Chama a atenção, nessa figura, a importância do câncer do estômago como causa básica de óbito, cujos coeficientes, com o avançar da idade, passam a ocupar a terceira posição. O câncer de útero, porção não especificada, ocupa, em geral, a quarta posição e seus coeficientes crescem, também, com a idade, com tendência semelhante à do câncer de colo uterino. Até a faixa etária de 30 a 34 anos, os coeficientes do câncer de ovário e de outros anexos do útero ocupam a quarta posição, porém, após os 35 anos, os coeficientes de câncer da traquéia, brônquios e pulmão passam a ocupar essa posição. 


\section{Tabela 4 - Coeficientes medianos de mortalidade por neoplasmas malignos dos órgãos respiratórios e intratorácicos, segundo os grupos etários e causas básicas (três dígitos), por 100.000 mulheres de 15 a 49 anos, no Estado de São Paulo, 1991 a 1995.}

\begin{tabular}{|c|c|c|c|c|c|c|c|}
\hline \multirow{2}{*}{$\begin{array}{l}\text { Código } \\
\text { CID-9 * }\end{array}$} & \multicolumn{7}{|c|}{ Idade } \\
\hline & 15 a 19 & 20 a 24 & 25 a 29 & 30 a 34 & 35 a 39 & 40 a 44 & 45 a 49 \\
\hline 160 & 0,00 & 0,00 & 0,00 & 0,07 & 0,00 & 0,00 & 0,12 \\
\hline$|6|$ & 0,00 & 0,00 & 0,00 & 0,07 & 0,16 & 0,50 & 0,52 \\
\hline 162 & 0,07 & 0,07 & 0,26 & 0,43 & $|, 3|$ & 3,00 & 5,32 \\
\hline 163 & 0,00 & 0,00 & 0,00 & 0,00 & 0,00 & 0,00 & 0,00 \\
\hline 164 & 0,06 & 0,06 & 0,07 & 0,07 & 0,08 & 0,13 & 0,13 \\
\hline 165 & 0,00 & 0,00 & 0,00 & 0,00 & 0,00 & 0,00 & 0,00 \\
\hline \multicolumn{8}{|c|}{$\begin{array}{l}160=\text { Neoplasma maligno das fossas nasais, ouvido médio e seios acessórios } \\
161=\text { Neoplasma maligno da laringe } \\
\text { 162 = Neoplasma maligno da traquéia, dos brônquios e do pulmão } \\
\text { 163= Neoplasma maligno da pleura } \\
\text { 164= Neoplasma malligno do timo, do coração e do mediastino } \\
165=\text { Neoplasma maligno de outras localizacões e de localizacões mal definidas }\end{array}$} \\
\hline
\end{tabular}

Com relação aos neoplasmas malignos do encéfalo, o coeficiente mediano, para todas as idades, no quinqüênio estudado, foi de 0,62 óbitos por 100.000 mulheres. Analisando-se os coeficientes medianos segundo os grupos etários, observamos que eles aumentaram gradativamente, com a idade, de 0,30, no grupo 15-19 anos, até 1,59 por 100.000 mulheres, no grupo 45-49 anos.

O câncer de tireóide, no total de mulheres de 15 a 49 anos, apresentou coeficiente mediano de apenas 0,06 por 100.000 muIheres no quinquênio estudado. Entretanto, comparando os coeficientes ano a ano, verificamos uma diminuição gradativa, de 0,13 em 1991 até apenas 0,02 por 100.000 mulheres em 1995. Com relação à idade, os coeficientes medianos do quinquênio se elevaram de 0,08 no grupo etário de 35 a 39 anos para 0,24 por 100.000 mulheres no grupo etário de 45 a 49 anos.

\section{Discussão}

As informações sobre mortalidade, obtidas pelos dados contidos nos atestados de óbito, têm as suas limitações, pois podem ocorrer imprecisões e falhas no preenchimento das declarações sobre as causas relacionadas ao evento. Entretanto, os estudos a partir desta fonte de dados são muito úteis, pois contribuem para um conhecimento aproximado das causas básicas de óbito, de como elas evoluem no tempo e de como se distribuem no espaço e de acordo com atributos individuais.

Os dados apresentados na Tabela I, para todos os agrupamentos relativos a neoplasmas da CID-9, mostram claramente que, nas mutheres de 15 a 49 anos, o agrupamento que apresentou o coeficiente mediano mais elevado do quinquênio foi o "Neoplasmas malignos dos ossos, do tecido conjuntivo, da pele e da mama", sendo 84,5\% deste agrupamento representado pelo câncer de mama. Observamos, também, na Tabela 5 e na Figura I, que os coeficientes medianos por câncer de mama aumentam intensamente com a idade a partir dos 30 anos de idade.

O câncer de mama éa principal causa de mortalidade feminina por neoplasmas nos países desenvolvidos e está se tornando, também, a primeira causa no nosso país, conforme as estatísticas de mortalidade do Ministério da Saúde 2 . Estas estatísticas, referentes ao período de 1980 a 1994, mostram uma tendência ascendente dos coeficientes padronizados por idade, para ambos os sexos, dos cânceres de mama, de próstata e de pulmão, uma tendência descendente do câncer de estômago e pouca variação para o câncer de colo do útero.

Os coeficientes de incidência, padronizados poridade, computados pelo Registro de Câncer de São Paulo", no período de 1969 a 1993 , mostram, neste município, para o câncer de mama, uma tendência crescente de 1969 a 1978, uma estabilização no período de 1978 a 1988 e um novo aumento no período de 1988 a 1993. Quanto ao câncer de estômago, esse estudo mostrou um aumento da incidência de 1969 1 1978 e depois uma diminuição gradativa, de 1978a 1993, emambos ossexos. jáo câncer de colo do útero, após um aumento da incidência de 1969a 1973, há uma pequena tendência dedecréscimo de 1973 a 1993. Com relaçãoàs leucemias, esse estudo revelou, tanto para a linfóide como paraa mielóide, uma levetendên- 


\section{Tabela 5 - Coeficientes medianos de mortalidade por neoplasmas malignos dos ossos, do tecido conjuntivo, da pele e da mama, segundo os} grupos etários e causas básicas (três dígitos), por 100.000 mulheres de 15 a 49 anos , no Estado de São Paulo, 1991 a 1995.

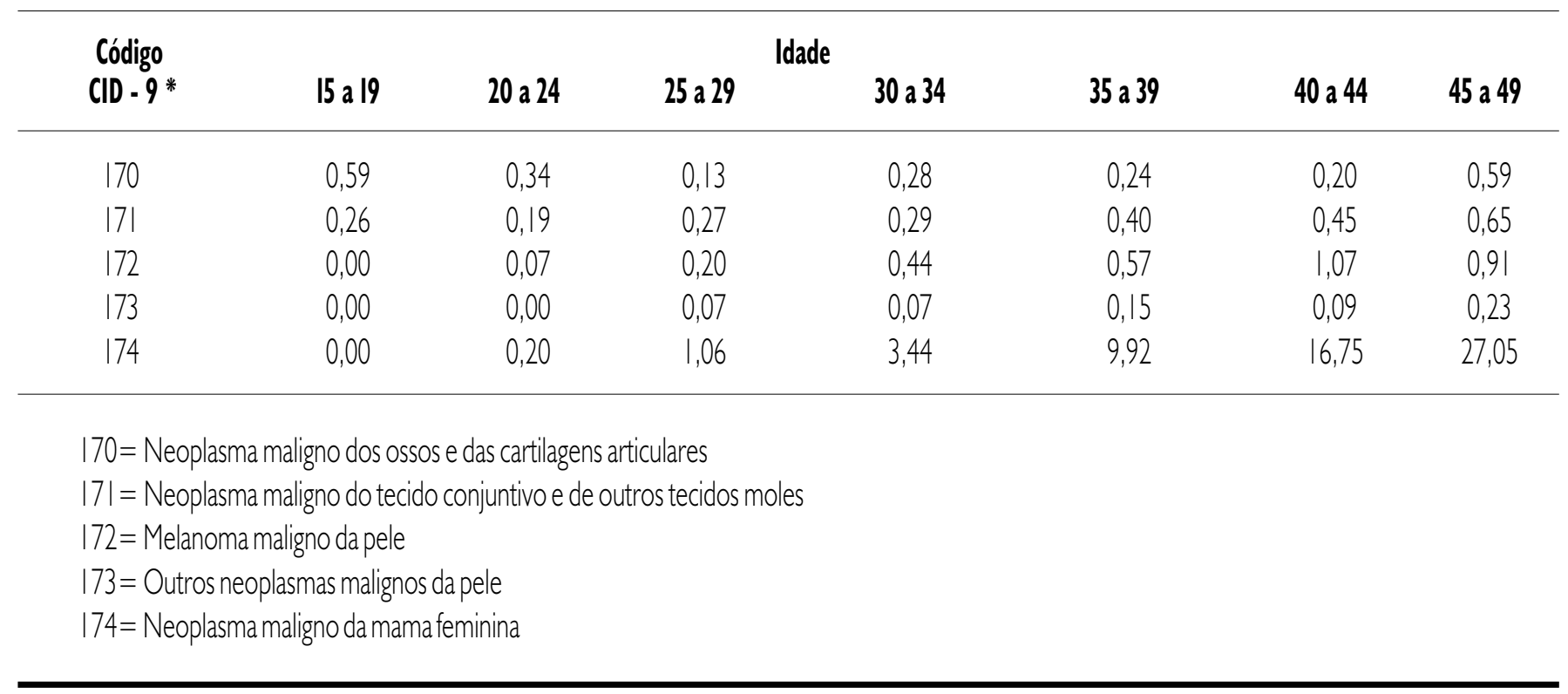

cia de elevação, de 1969 a 1993, nos dois sexos. Os coeficientes de câncer de pulmão, mais altos no sexo masculino do que no feminino, apresentaram, em ambos os sexos, uma tendência paulatina de ascenção no período estudado.

Em nosso estudo, o câncer de colo uterino e o câncer de útero, porção não especificada, o qual provavelmente deve estar em grande parte relacionado ao primeiro, estão em segundo lugar na ordem decrescente dos coeficientes, demonstrando que no Estado de São Paulo ainda o problema persiste, traduzindo insuficiência das atividades preventivas como a educação sanitária e a realização periódica de exames de citologia vaginal e colposcopia ${ }^{13}$. Lopes et al. ${ }^{14}$, estudando o comportamento da população feminina brasileira referente à prevenção do câncer de colo uterino, verificaram que $76 \%$ das mulheres tinham conhecimento do teste de Papanicolau, porém $64 \%$ fizeram o teste apenas uma vez e somente $30 \%$ o fizeram mais de três vezes.

Com relação aos neoplasmas de órgãos digestivos, o presente estudo mostrou maiores coeficientes para o câncer de estômago, principalmenteapós os 30 anos de idade, seguido dos cânceres do cólon e dofigado evias biliares intrahepáticas (Tabela 3 e Figura I).

Embora as estatísticas de mortalidade e de incidência tenham mostrado uma tendência de diminuição do câncer de estômago nas últimas décadas em vários países e mesmo no Brasil|' ${ }^{\prime \prime}$, ele continua sendo uma causa importante de mortalidade, também em mulheres relativamente jovens, conforme os nossos dados demonstram.

Em nosso estudo, dentre as formas de câncer dos órgãos respiratórios e intra-torácicos, o neoplasma maligno da traquéia, dos brônquios e do pulmão teve os coeficientes mais elevados, ocupando a quinta posição, após os 35 anos, entre as principais causas específicas de óbito por câncer (Tabela 4 e Figura I). O aumento gradativo dos coeficientes de câncer de pulmão, revelado em dados de mortalidade no Brasil ${ }^{2}$ e de incidência no município de São Paulo "', podem estar relacionados com o aumento da proporção de fumantes entre mulheres jovens.
Com relação à diminuição dos coeficientes de mortalidade por câncer de tireóide, de 1991 a 1995, vale ressaltar que os coeficientes de incidência para o Município de São Paulo se elevaram nas mulheres dos grupos etários 20-24, 25-29, 30-34, 35-39, 40-44 e 45-49, nos anos de 1983 para 1988 e para 1993'I. Se esse aumento de incidência também tivesse ocorrido em todo o Estado de São Paulo, a explicação da diminuição da mortalidade observada no nosso estudo talvez pudesse ser explicada por diagnósticos mais precoces, pelo uso de ultra-sonografia e punção-biópsia de nódulos da tireóide (que aumentariam a incidência), proporcionando pronto tratamento de formas iniciais do câncer dessa glândula, levando à cura ou maior sobrevida.

Os dados apresentados neste trabalho mostram a necessidade de maiores estudos epidemiológicos para investigar fatores de risco relativos aos vários tipos de câncer, em especial os que causam maior incidência e mortalidade, como os cânceres de mama, de colo uterino e de estômago. Medidas preventivas devem ser 
Tabela 6 - Coeficientes medianos de mortalidade por neoplasmas malignos dos órgãos genitourinários, segundo os grupos etários e causas básicas (três dígitos), por 100.000 mulheres de 15 a 49 anos, no Estado de São Paulo, 199 I a 1995.

\begin{tabular}{|c|c|c|c|c|c|c|c|}
\hline \multirow{2}{*}{$\begin{array}{c}\text { Código } \\
\text { CID - } 9 \text { * }\end{array}$} & \multicolumn{7}{|c|}{ Idade } \\
\hline & 15 a 19 & 20 a 24 & 25 a 29 & 30 a 34 & 35 a 39 & 40 a 44 & 45 a 49 \\
\hline 179 & 0,00 & 0,06 & 0,27 & 0,81 & 1,86 & 3,49 & 5,79 \\
\hline 180 & 0,00 & 0,14 & 0,66 & 1,83 & 3,50 & 6,44 & 10,56 \\
\hline 181 & 0,00 & 0,00 & 0,07 & 0,07 & 0,08 & 0,00 & 0,00 \\
\hline 182 & 0,00 & 0,00 & 0,00 & 0,07 & 0,08 & 0,32 & 0,54 \\
\hline 183 & 0,33 & 0,20 & 0,41 & 0,62 & $|, 3|$ & 2,88 & 4,98 \\
\hline 184 & 0,00 & 0,00 & 0,07 & 0,07 & 0,16 & 0,21 & 0,83 \\
\hline 188 & 0,00 & 0,00 & 0,00 & 0,00 & 0,08 & 0,18 & 0,39 \\
\hline 189 & 0,00 & 0,07 & 0,13 & 0,21 & 0,33 & 0,40 & 0,68 \\
\hline \multicolumn{8}{|c|}{$\begin{array}{l}179=\text { Neoplasma maligno do útero, porção não especificada } \\
\text { 180 = Neoplasma maligno do colo do útero } \\
\text { 181 = Neoplasma maligno da placenta } \\
\text { 182 = Neoplasma maligno do corpo do útero } \\
\text { 183 = Neoplasma maligno do ovário e de outros anexos do útero } \\
\text { 184 = Neoplasma maligno de outros órgãos genitais femininos e dos não especificados } \\
\text { 188= Neoplasma maligno da bexiga urinária } \\
189=\text { Neoplasma maligno do rim, de outros órgãos urinários e de órgãos urinários não especificados }\end{array}$} \\
\hline
\end{tabular}

Tabela 7 - Coeficientes medianos de mortalidade por neoplasmas malignos dos tecidos linfático e hematopoiético, segundo os grupos etários e causas básicas (três dígitos), por 100.000 mulheres de I5 a 49 anos, no Estado de São Paulo, I99I a 1995.

\begin{tabular}{|c|c|c|c|c|c|c|c|}
\hline \multirow{2}{*}{$\begin{array}{l}\text { Código } \\
\text { CID - } 9 *\end{array}$} & \multicolumn{7}{|c|}{ Idade } \\
\hline & 15 a 19 & 20 a 24 & 25 a 29 & 30 a 34 & 35 a 39 & 40 a 44 & 45 a 49 \\
\hline 200 & 0,12 & 0,00 & 0,07 & 0,07 & 0,08 & 0,20 & 0,13 \\
\hline 201 & 0,14 & 0,19 & 0,27 & 0,37 & 0,34 & 0,40 & 0,41 \\
\hline 202 & 0,25 & 0,33 & 0,33 & 0,50 & 0,80 & 0,97 & 1,49 \\
\hline 203 & 0,00 & 0,00 & 0,00 & 0,14 & 0,17 & 0,58 & 0,95 \\
\hline 204 & 0,59 & 0,39 & 0,33 & 0,14 & 0,32 & 0,30 & 0,52 \\
\hline 205 & 0,58 & 0,80 & 0,99 & 0,97 & 1,18 & 1,68 & 2,11 \\
\hline 208 & 0,18 & 0,13 & 0,20 & 0,14 & 0,23 & 0,32 & 0,41 \\
\hline \multicolumn{8}{|c|}{$\begin{array}{l}200=\text { Linfossarcoma e reticulossarcoma } \\
201 \text { = Doença de Hodgkin } \\
202 \text { = Outros neoplasmas malignos dos tecidos linfóide e histiocitário } \\
203 \text { = Mieloma múltiplo eneoplasmas imunoproliferativos } \\
204=\text { Leucemia linfóide } \\
205 \text { = Leucemia mielóide } \\
208=\text { Leucemia de tipo celular não especificado } \\
\text { Obs. : As categorias } 206 \text { e } 207 \text { não são apresentadas devido à sua frequêencia muito baixa ou nula nos vários grupos etários. }\end{array}$} \\
\hline
\end{tabular}


Figura I - Coeficientes medianos de mortalidade por neoplasmas malignos, segundo as principais causas básicas (três dígitos) e grupos etários, por 100.00 mulheres de I5 a 49 anos, no Estado de São Paulo, 199 I a 1995.

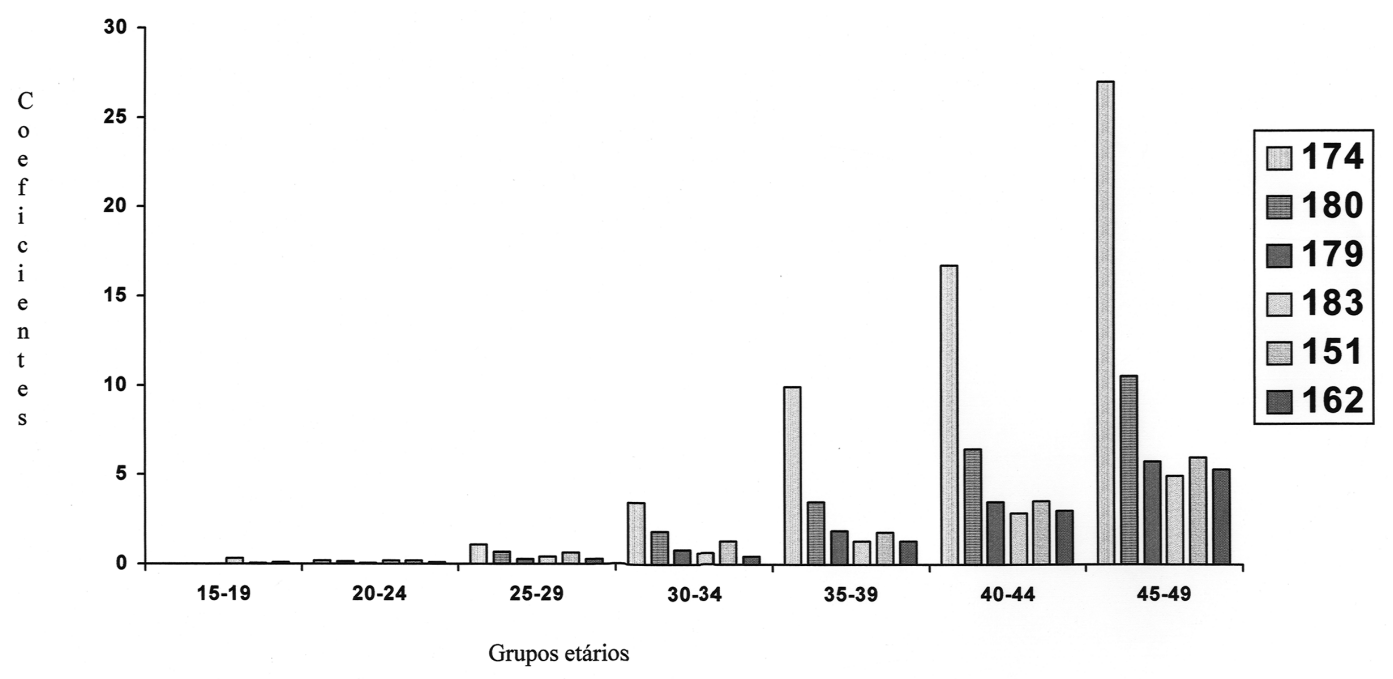

$174=$ Câncer de mama $\quad 180=$ Câncer do colo do útero $\quad 179=$ Câncer do útero, porção não especificada 183 = Câncer de ovário e de outros anexos do útero $\quad 151$ = Câncer do estômago $162=$ Câncer da traquéia, brônquios e pulmão

intensificadas, visandoà diminuição da exposição aos fatores de risco conhecidos (como o hábito de fumar, doenças sexualmente transmissíveis, hábitos alimentares inadequados, etc.) bem como a organização efuncionamento adequado de senviços de saúde dedicados ao diagnóstico e tratamento precoce dos neoplasmas malignos em mulheres em idade fértil, não só para preservar a saúde e prolongar a vida, como também para evitar possíveis futuras gestações complicadas com essas doenças.

\section{Agradecimento}

À Fundação Sistema Estadual de Análise de Dados (SEADE) pelos dados fornecidos.

\section{SUMMARY}

MORTALITY OWING TO NEOPLASMS IN WOMEN DURING THE REPRODUCTIVE AGE (I5 to 49 years) in the state of São Paulo, BRAZIL, FROM 1991 TO 1995.

OBJECTIVE. To describe the mortality owing to neoplasms in women of reproductive age ( 15 to 49 years) in the State of
São Paulo, Brazil, from $199 /$ to 1995.

METHODS. A list of all deaths and their underlying causes, coded according to the International Classification of Diseases, $9^{\text {th }}$ revision, and estimates of the female population according to age groups were provided by the SEADE Foundation. Specific coefficients for 100 thousand women for each year as well as the medians of the coefficients related to 5 years, and the percentage of underlying causes of death by subgroups were calculated.

RESULTS. In young women (15 to 29 years) highest rates were found for the subgroup "Malignant neoplasm of lymphatic and hematopoiethic tissues", followed by the subgroup "Malignant neoplasm of bones, connective tissue, skin and breast". In women aged 30 to 49 years, the leading underlying causes of death were those included in the last subgroup, followed by the subgroups "Malignant neoplasm of genitourinary organs", "Malignant neoplasm of digestive organs and peritoneum", and "Malignant neoplasm of lymphatic and hematopoietic tissues". The highest specific rates of underlying causes of death for women aged 15-29 were myeloid and lymphoid leukemias, and for women aged 30-49 were in decreasing rank," breast cancer"; "uterine cervix cancer" and "not specified uterine part cancer"; "stomach cancer", "tracheal, bronchial and lung cancer" and "ovarian and other uterine annexes".

Conclusion. Preventive measures to eliminate or lower the risk factors exposure and to promote early diagnosis and prompt treatment of these neoplasms should be enhanced in order to avoid possible future pregnancy complications and to prolong survival of women of reproductive age. [Rev Ass Med Brasil 200 ; 47(3): 221-30]

KEY WoRDs: Female mortality of reproductive age. Neoplasms. Breast cancer. Uterine cancer. Stomach cancer. Leukemia.

\section{ReferênCIAS}

I. Lotufo PA. Non-communicable diseases in Brazil: mortality patterns, morbidity studies 
HADDAD N ET AL.

and risk factors. Arch Lat Nutrición 1997; 47:25-9.

2. Ministério da Saúde. Instituto Nacional do Câncer. Coordenação de Programas de Controle do Câncer. O problema do câncer no Brasil. 4a ed. Rio de Janeiro, 1997.

3. Carvalheiro CDG. Estudo de algumas variáveis associadas à população feminina em idade fértil [tese]. Ribeirão Preto: Faculdade de Medicina de Ribeirão Preto da Universidade de São Pauo; 1975.

4. Carvalheiro CDG, Manço ARX. Mortalidade feminina no período reprodutivo em localidade urbana da região sudeste do Brasil. Evolução nos últimos 20 anos. Rev Saúde Pública 1992; 26:239-45.

5. Laurenti R, Buchalla CM, Lolio CA, Santo AH, Mello Jorge MHP. Mortalidade de mulheres em idade fértil no Município de São Paulo (Brasil), 1986: I- Metodologia e resultados gerais. Rev Saúde Pública 1990; 24: 128-33.

6. Haddad N, Silva MB. Mortalidade feminina em idade reprodutiva no Estado de São Paulo, Brasil, 199|-1995: causas básicas de óbito e mortalidade materna. Rev Saúde Pública 2000; 34:64-70.

7. Haddad N, Silva MB. Mortalidade por doenças cardiovasculares em mulheres em idade reprodutiva ( 15 a 49 anos), no Estado de São Paulo, Brasil, 1991 a 1995. Arq Bras Cardiol 2000; 75:369-74.

8. Organização Mundial da Saúde. Manual da classificação estatística internacional de doenças, lesões e causas de óbito, 9a Rev. 1975. São Paulo: Centro da OMS para Classificação de Doenças em Português, 1980.

9. Giraldelli BW, Saad PM. Considerações sobre a implantação no Estado de São Paulo do Sistema Automático de Classificação de Causas de Morte. Inf Demogr 1984; 13: I37-59.

10. Norusis MJ. SPSS for Windows: Release 6.0. Chicago, SPSS Inc, 1993.

II. Ministério da Saúde. Instituto Nacional do Câncer. Faculdade de Saúde Pública da USP.
Registro de Câncer de São Paulo. Incidência de câncer no Município de São Paulo, Brasil. Tendência no período 1969-1993. Brasília, DF, Ministério da Saúde, 1999.

12. Restrepo HE. Epidemiología y control del cáncer de la mujer en América Latina y el Caribe. In: Organización Panamericana de la Salud. Género, mujer y salud en las Américas. Pub. Cient. n54I. Washington, D.C. 1993; 98-II3.

13. Mendonça GAS. Câncer na população feminina brasileira. Rev Saúde Pública 1993; 27: 68-75.

14. Lopes ER, Rebelo MS, Abreu E, Silva E, Costa VL, Eisenberg ALM et al. Comportamento da população brasileira feminina em relação ao câncer cérvico-uterino. J Bras Ginecol 1995; 105:505-16.

Artigo recebido: 08/12/2000 Aceito para publicação: |4/06/200 |

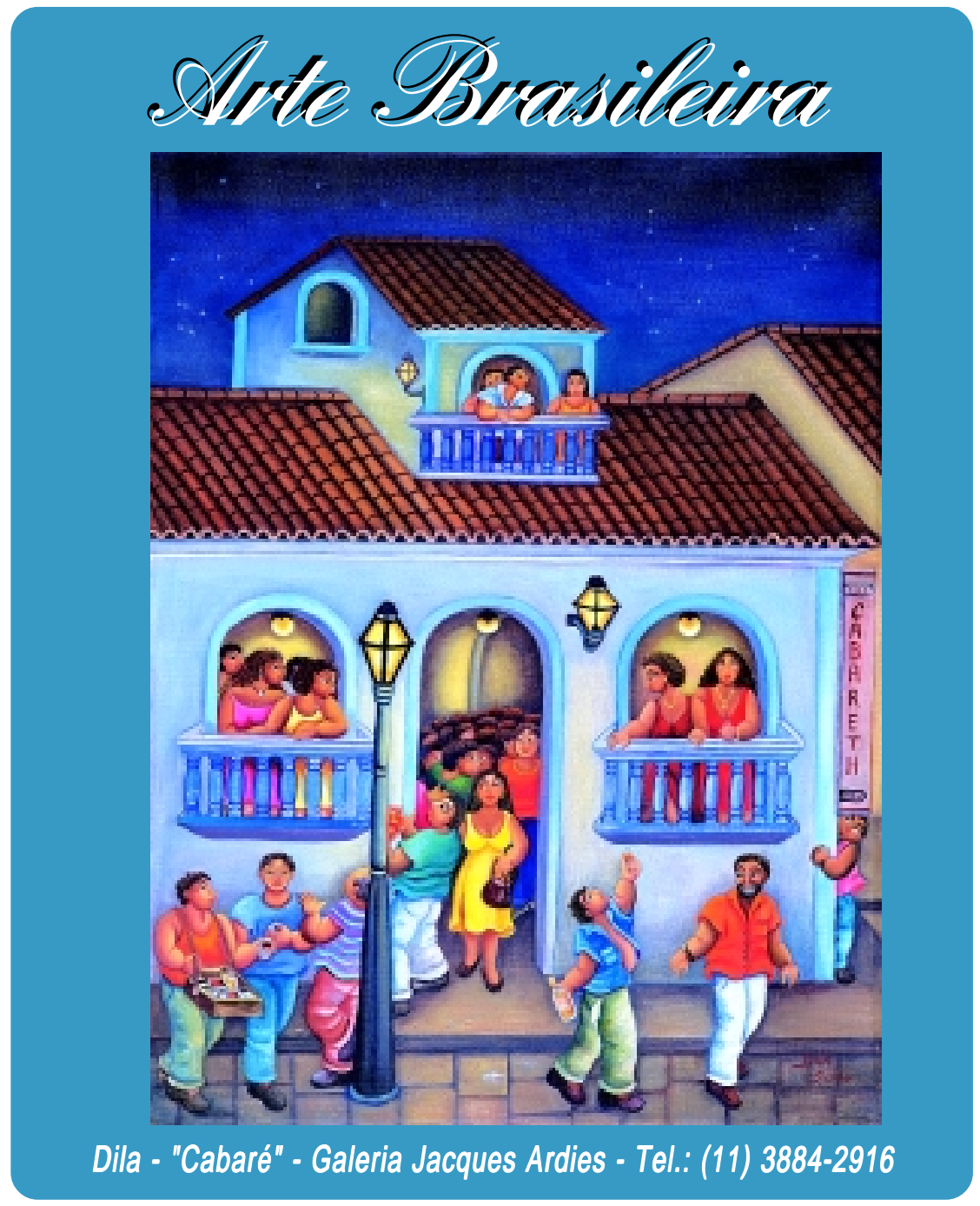

